\title{
Inhibition of Colorectal Cancer Cells HT-29 by Secretome and Extraction of Human Umbilical Cord Wharton's Jelly Stem Cells
}

Mahdieh Farhoudi Sefidan Jadid

Islamic Azad University

Parisa Emami

Islamic Azad University

Pouya Goleij

Sana Institute of Higher Education

Vahidreza Karamad

Ege University

Afshin Khorrami

Yazd University

Gholamreza Jahangirzadeh

Islamic Azad University

Mona Moghadami

Babol University of Medical Sciences

Alireza Isazadeh

Tabriz University of Medical Sciences

Saba Hajazimian ( $\sim$ hajazimian.saba@gmail.com )

Tabriz University of Medical Sciences

\section{Research Article}

Keywords: Stem cells, Wharton's jelly, Colorectal cancer, Apoptosis

Posted Date: January 9th, 2021

DOl: https://doi.org/10.21203/rs.3.rs-142535/v1

License: (c) (i) This work is licensed under a Creative Commons Attribution 4.0 International License.

Read Full License 


\section{Abstract}

Background and aim: Colorectal cancer (CC) is aggressive cancer and the major cause of death worldwide that need the development of novel and effective therapeutic methods. Recently suggested that the human Wharton's jelly stem cells (hWJSCs) have an anti-proliferation activity against of several cancer cells through apoptosis induction. Therefore, this study aimed to investigate the effects of conditioned medium and cell lysate of human umbilical cord hWJSCs against the HT-29 cancer cell line and mechanisms of apoptosis induction.

Methods: In this study, the hWJSCs conditioned medium and cell lysate were prepared from 10 human umbilical cord samples. The effects of hWJSCs conditioned medium and cell lysate were evaluated on the viability, migration, invasion, and apoptosis of HT-29 CC cell line. The expression of apoptosis related $B A X, B C L 2$, SMAC, SURVIVIN, and Cas9 genes were evaluated in CC cells treated with hWJSCs conditioned medium and cell lysate.

Results: We observed that conditioned medium and cell lysate of hWJSCs decrease CC cells viability, proliferation, migration, and invasion in a concentration- and time-dependent manner. Moreover, conditioned medium and cell lysate increased apoptosis rate of CC cells, which can be due to increase BAX, SMAC, and Cas9 genes, as well as decrease BCL2 and SURVIVIN genes.

Conclusions: Our study suggest that conditioned medium and cell lysate of hWJSCs can inhibit CC cells through induction of apoptosis. However, further studies on are required to more accurate results.

\section{Introduction}

Colorectal cancer (CC) is a common cancer and cause of death in worldwide [1]. The chemotherapy is one of the most important method to treatment of patient with CC. However, resistance of cancer cells to various chemotherapeutic agents cause to limited efficacy of CC chemotherapy [2]. Therefore, use of novel therapeutic methods is required in treatment of patients with CC [3]. Recently, many studies have reported the appropriate effects of stem cells in suppress, management, and treatment of various cancers in digestive system $[3,4]$.

The mesenchymal stem cells (MSCs) have shown an anticancer effect against several cancer cells. Therefore, the MSCs considered as notable anticancer compound to inhibition of several human cancer cells [5]. The human Wharton's jelly stem cells (hWJSCs) are an important type of MSCs that have been isolated from gelatinous Wharton's jelly of human umbilical cord [6, 7]. The evidences suggested that hWJSCs have regenerative capacity more than other autologous MSCs of the adults $[8,9]$. Recently, several studies have suggested that hWJSCs presents a notable anti-proliferative effects against several cancer cells, such as ovarian carcinoma, mammary adenocarcinoma, osteosarcoma, bladder carcinoma, lymphoma, and cholangiocarcinoma $[10,11]$. 
Today, the underlying mechanisms of hWJSCs antitumor activity have not been identified. Although some studies suggested that hWJSCs inhibits the cancer cells proliferation through apoptosis induction $[9,11]$. Therefore, the present study aimed to investigate the inhibitory effects of secretome and extraction of hWJSCs against CC cells, and underlying mechanisms of anticancer activity and apoptosis induction in the HT-29 cancer cell line.

\section{Materials And Methods}

\section{Isolation and culture of hWJSCs}

In present study, 10 human umbilical cords were received from pregnant women referred to Shahryar Hospital, Tabriz, Iran. The umbilical cords were transferred Biotechnology Research Center, Islamic Azad University, Tabriz branch, in proximity of the ice. Initially, the Wharton's jelly tissues were primary cultured (explant culture) using complete Dulbecco's modified eagle medium (DMEM) contains $1 \%$ penicillinstreptomycin antibiotic and $10 \%$ fetal bovine serum (FBS) at $37^{\circ} \mathrm{C}$ and $5 \% \mathrm{CO}_{2}$ (9). It is noteworthy that all selected women were signed a consent form according to the ethical standards of Helsinki Declaration.

\section{Preparation of conditioned medium and cell lysate of hWJSCs}

The isolated hWJSCs were cultured using DMEM medium contains $1 \%$ penicillin-streptomycin (5000 units $/ \mathrm{mL}-5000 \mathrm{mg} / \mathrm{mL}$ ) antibiotics without FBS at $37^{\circ} \mathrm{C}$ and $5 \% \mathrm{CO} 2$ for 72 hours. Next, supernatant medium was collected as conditioned medium, and sterilized using a $0.22 \mu \mathrm{m}$ filter. Then, the cell lysis buffer (150 mM NaCl, 1.0\% Nonyl Phenoxypolyethoxylethanol-NP40, $50 \mathrm{mM}$ Tris-Cl, 1.0\% sodium deoxycholate, $0.1 \%$ SDS) and protease inhibitor were added to the cultured cells. The obtained cell suspension was centrifuged and cells pellet was collected as cell lysate.

\section{Cancer cells culture}

The HT-29 cancer cells were purchased from cell bank of Immunology Research Center (IRC), Tabriz University of Medical Sciences. The obtained cancer cells were cultured using Roswell Park Memorial Institute (RPMI) 1640 medium containing 10\% FBS and 1\% penicillin-streptomycin at standard condition.

\section{Cancer cells viability assay}

The CC cells $\left(1 \times 10^{4} /\right.$ well) were seeded in 96 -well plate containing culture medium supplemented by FBS. After 24 hours, the cancer cells were treated with conditioned medium $(38,40,42,44$, and $46 \%)$ and cell 
lysate $(11,12,13,14$, and 15\%), and incubated for 72 hours. The Tetrazolium Micro-culture (MTT) method was performed according to the manufacturer's instruction. For this purpose, the old culture medium was replaced with RPMI medium containing MTT agent solution (50 $\mu \mathrm{l}$ ) in phosphate-buffered saline (PBS), and incubated for 4 hours. Next, the old culture medium replaced with $50 \mu \mathrm{L}$ Dimethyl sulfoxide (DMSO) and incubated for 30 minutes. The absorbance of wells was measured using enzyme-linked immunosorbent assay (ELISA) reader at $540 \mathrm{~nm}$. Finally, the treated cancer cells viability was measured by following formula [(treated sample OD / untreated sample OD) $\times 100]$.

\section{Transwell invasion assay}

The CC cells were seeded in the upper chamber of transwell chamber in RPMI medium (serum-free), and treated by conditioned medium (44\%) and cell lysate (14\%). Moreover, the complete medium was added to the bottom chamber and incubated for 20 hours. Then the migrated cancer cells in bottom chamber were fixed by paraformaldehyde (4\%) and stained by crystal violet $(0.1 \%)$. Finally, the migrated cancer cells were evaluated by inverted phase contrast microscopy.

\section{Wound-healing migration assay}

The CC cells $\left(1 \times 10^{4} /\right.$ well) were seeded in 96 -well plate containing culture medium supplemented by FBS. The monolayer cancer cells were scratched by pipette tips and washed with PBS. Then, cancer cells were treated with conditioned medium (44\%) and cell lysate (14\%), and incubated for 72 hours for 20 hours. Finally, the migrated cancer cells were evaluated by inverted phase contrast microscopy.

\section{Cancer cells apoptosis assay}

The CC cells $\left(1 \times 10^{4} /\right.$ well) were seeded in 96 -well plate containing culture medium supplemented by FBS. After 24 hours, the cancer cells were treated with conditioned medium (44\%) and cell lysate (14\%), and incubated for 72 hours. The CC cells were washed, trypsinized, and resuspended in binding buffer with propidium iodideand annexin $\mathrm{V}$. Then, cancer cells suspension incubated at dark place and room temperature with Annexin V-FITC $(5 \mathrm{~mL}), \mathrm{PI}(10 \mathrm{~mL})$, and binding buffer $(400 \mu \mathrm{L})$ for 15 minutes. Finally, quantitative apoptosis was measured using fluorescein isothiocyanate (FITC)/PI by flowcytometry instrument.

\section{Quantification of apoptosis-related genes expression}


The CC cells $\left(1 \times 10^{4} /\right.$ well) were seeded in 96 -well plate containing culture medium supplemented by FBS. After 24 hours, the cancer cells were treated with conditioned medium (44\%) and cell lysate (14\%), and incubated for 48 hours. Extraction of total RNA was performed by Trizol reagent, and cDNA synthesized using reverse transcription. The mRNA expression of BAX, BCL2, SMAC, SURVIVIN, and Cas9 genes were evaluated using quantitative Real Time PCR and specific primers (Table 1). The Real Time PCR analysis was performed using SYBR green master mix and relative expression was evaluated using comparative CT $\left(2^{-\Delta \Delta C T}\right)$ method. The $\beta$-actin gene was considered as endogenous control.

\section{Statistical analysis}

The statistical analysis between treatment and control cells were performed by one-way ANOVA and Tukey (post-hoc) analysis. The results were presented as mean \pm standard deviation (SD) from three replicates, and $p<0.05$ was considered statistically significant.

\section{Results}

\section{Cancer cells viability}

Our study showed that the CC cells viability decreased as time and concentration dependent manner after treatment with conditioned medium $(38,40,42,44$, and $46 \%)$ and cell lysate $(11,12,13,14$, and $15 \%)$. The viability of cancer cells significantly decreased following treatment with high concentrations of conditioned medium and cell lysate (Figure 1). We observed that the anti-proliferation activity of cell lysate was significantly more than conditioned medium. The half maximal inhibitory concentration (IC50) of conditioned medium and cell lysate on CC cells after 48 hours were $44 \%$ and $14 \%$, respectively.

\section{Cancer cells migration and invasion}

The effects of hWJSCs conditioned medium and cell lysate on invasion and migration of CC cells were evaluated with wound healing assay and transwell migration assay. The results of wound healing assay and transwell migration assay showed that the migration of CC cells significantly decreased after treatment with conditioned medium (44\%) and cell lysate (14\%) (Figure 2, Figure 3).

\section{Cancer cells apoptosis}

The quantification of apoptosis assay showed that the early and late apoptosis rate significantly increased in CC cells following treatment with conditioned medium (44\%) and cell lysate (14\%). We 
observed that the apoptosis induction by cell lysate was significantly more than conditioned medium (Figure 4).

\section{Expression of apoptosis related genes}

The evaluation of apoptosis related genes expression showed that the conditioned medium (44\%) and cell lysate (14\%) significantly increased mRNA expression of BAX, SMAC and Cas 9 genes (Figure 5), as well as significantly decreased mRNA expression of BCL2 and SURVIVIN genes (Figure 5). We observed that regulation of apoptosis related genes expression in CC cells by cell lysate was significantly more than conditioned medium.

\section{Discussion}

Inhibition of apoptosis is an important pathological processes in patients with CC, which caused by induction of anti-apoptotic genes as well as suppression of pre-apoptotic genes expression [12]. The dysregulated proliferation of cancer cells and resistance to apoptosis are two important causes of cancer progression [13]. Therefore, induction of apoptosis by various natural and chemical compounds is considered as an anticancer strategy $[14,15]$. In this regards, several studies reported that hWJSCs have an appropriate anticancer activity through various mechanisms $[5,9]$. This stem cells are easily derived from umbilical cord and suggested as an anticancer agent $[16,17]$. The hWJSCs presents several advantages as compared to other stem cells, and are derived from human umbilical cord which are discarded at birth. Moreover, the hWJSCs are highly proliferative and widely multipotent with long telomeres [7].

In present study, we investigated viability and proliferation of HT-29 cancer cells, and underlying mechanisms of apoptosis induction following treatment by hWJSCs conditioned medium and cell lysate. We observed that conditioned medium and cell lysate significantly decreased viability and proliferation of CC cancer cells. In a recent study by Kalamegam et al. reported that cell lysate of hWJSCs inhibits cellular growth and proliferation, as well as apoptosis in ovarian cancer cells [18]. Moreover, Han et al. reported that MSCs derived from human cord inhibits cellular growth and proliferation of prostate cancer cells [19].

The metastasis and invasion in patients with CC is the most cause of death [20]. Therefore, we evaluated the effects of conditioned medium and cell lysate on inhibition of metastasis and invasion of CC cells using transwell migration assays and wound healing assay. Our study showed that the invasion and migration of CC cells were significantly inhibited following treatment with conditioned medium and cell lysate. In a most recent study by Hajazimian et al. (2020) reported that conditioned medium and cell lysate inhibited endometriosis cells migration and invasion through several molecular pathways, as well as inhibition of MMP-2 and MMP-9 genes expression [9]. Previous studies demonstrated that the MMP-2 
and MMP-9 genes play important role in metastasis and invasion of various cancer cells [21,22]. Both MMP-2 and MMP-9 genes are overexpressed in approximately all human cancer cells [23].

Induction of apoptosis is an important approach for cancer cell death. The evidence suggested that the hWJSCs conditioned medium and cell lysate cause to induction of apoptosis in several cancer cells [5]. Therefore, the apoptosis rate of CC cells was investigated by Annexin V-FITC/PI method following treatment by conditioned medium and cell lysate. Our study showed that the hWJSCs conditioned medium and cell lysate significantly increased CC cells apoptosis. The intrinsic and mitochondrial apoptosis pathways are usually regulated through modification of several genes family members [24]. The evidence suggested that upregulation of BAX, SMAC, and Cas9 genes, as well as downregulation of $B C L-2$ and SURVIVIN genes causes to apoptosis onset [24, 25]. In this regards, we showed that conditioned medium and cell lysate significantly upregulated $B A X, S M A C$, and Cas9 genes expression, as well as downregulated $B C L-2$ and SURVIVIN genes expression in CC cells. These results suggested the effects of conditioned medium and cell lysate on induction of intrinsic apoptotic pathway in HT-29 CC cells.

\section{Conclusion}

Generally, we suggested that the hWJSCs conditioned medium and cell lysate stimulates of immune system, and also prevent the viability, proliferation, metastasis, and invasion of CC cells. We showed that hWJSCs conditioned medium and cell lysate cause to induction of intrinsic apoptotic pathway in CC cells. Therefore, the hWJSC can be used in the future to control and even treatment of patients with CC. However, further studies are necessary to explain the exact effects of hWJSCs on physiopathology of CC.

\section{References}

1. Haggar FA, Boushey RP. Colorectal cancer epidemiology: incidence, mortality, survival, and risk factors. Clin Colon Rectal Surg. 2009;22(04):191-7.

2. Taheri E, Ghorbani S, Safi M, Sani NS, Amoodizaj FF, Hajazimian S, Heidari M, Isazadeh A, Heidari M. Inhibition of Colorectal Cancer Cell Line CaCo-2 by Essential Oil of Eucalyptus camaldulensis Through Induction of Apoptosis. Acta Med Iran 2020;58(6):260-265.

3. Soheilyfar S, Velashjerdi Z, Hajizadeh YS, Maroufi NF, Amini Z, Khorrami A, Azimian SH, Isazadeh A, Taefehshokr S, Taefehshokr N. In vivo and in vitro impact of miR-31 and miR-143 on the suppression of metastasis and invasion in breast cancer. J BUON. 2018;23(5):1290-6.

4. Ohigashi S, Hoshino Y, Ohde S, Onodera H. Functional outcome, quality of life, and efficacy of probiotics in postoperative patients with colorectal cancer. Surg Today. 2011;41(9):1200-07.

5. Gauthaman K, Yee FC, Cheyyatraivendran S, Biswas A, Choolani M, Bongso A. Human umbilical cord Wharton's jelly stem cell (hWJSC) extracts inhibit cancer cell growth in vitro. J Cell Biochem. 2012;113(6):2027-39. 
6. Lin DH, Biswas A, Choolani M, Fong CY, Bongso A. Induction of immunogenic cell death in lymphoma cells by Wharton's jelly mesenchymal stem cell conditioned medium. Stem Cell Rev Rep. 2017;13(6):801-16.

7. Fong CY, Subramanian A, Biswas A, Gauthaman K, Srikanth P, Hande MP, Bongso A. Derivation efficiency, cell proliferation, freeze-thaw survival, stem-cell properties and differentiation of human Wharton's jelly stem cells. Reprod Biomed Online. 2010;21(3):391-401.

8. Kamal MM, Kassem DH. Therapeutic potential of wharton's jelly mesenchymal stem cells for diabetes: achievements and challenges. Front Cell Dev Biol. 2020;8.

9. Hajazimian S, Maleki M, Danaei Mehrabad S, Isazadeh A. Human Wharton's Jelly Stem Cell Inhibit Endometriosis through Apoptosis Induction. Reproduction. 2020;159(5):549-558.

10. Tamura M, Kawabata A, Ohta N, Uppalapati L, G Becker K, Troyer D. Wharton's jelly stem cells as agents for cancer therapy. J Tissue Eng Regen Med. 2011;4(1):39-47.

11. Hendijani F, Javanmard SH. Dual protective and cytotoxic benefits of mesenchymal stem cell therapy in combination with chemotherapy/radiotherapy for cancer patients. Crit Rev Eukaryot Gene Expr. 2015;25(3):203-207.

12. Maroufi NF, Vahedian V, Hemati S, Rashidi M, Akbarzadeh M, Zahedi M, et al. Targeting Cancer Stem Cells by Melatonin: Effective Therapy for Cancer Treatment. Pathol Res Pract. 2020;152919.

13. Vahedian V, Asadi A, Esmaeili P, Zamani S, Zamani R, Hajazimian S, et al. Anti-inflammatory activity of emu oil-based nanofibrous scaffold through downregulation of IL-1, IL-6, and TNF-a proinflammatory cytokines. Horm Mol Biol Clin Investig. 2020;20190052.

14. Fatemeh Firouzi Amoodizaj, Sevda Baghaeifar, Elham Taheri, Mahdieh Farhoudi, Maryam Safi, Saba Hajazimian, Alireza Isazadeh, Dariush Shanehbandi*. Enhanced Anticancer Potency by Combination Chemotherapy of Gastric Cancer Cells Line AGS by Doxorubicin and Curcumin. J Biochem Mol Toxicol. 2020;e22486.

15. Maroufi NF, Vahedian V, Akbarzadeh M, Mohammadian M, Zahedi M, Isazadeh A, Pouremamali $F$, Taefehshokr S, Heidari M, Rashidi M, Nouri M. The apatinib inhibits breast cancer cell line MDA-MB231 in vitro by inducing apoptosis, cell cycle arrest, and regulating nuclear factor-KB (NF-KB) and mitogen-activated protein kinase (MAPK) signaling pathways. Breast Cancer. 2020;1-8.

16. Yuan Z, Kolluri KK, Gowers KH, Janes SM. TRAIL delivery by MSC-derived extracellular vesicles is an effective anticancer therapy. J Extracell Vesicles. 2017;6(1):1265291.

17. Liu B, Chen F, Wu Y, Wang X, Feng M, Li Z, Zhou M, Wang Y, Wu L, Liu X, Liang D. Enhanced tumor growth inhibition by mesenchymal stem cells derived from iPSCs with targeted integration of interleukin24 into rDNA loci. Oncotarget. 2017;8(25):40791.

18. Kalamegam G, Sait KH, Ahmed F, Kadam R, Pushparaj PN, Anfinan N, Rasool M, Jamal MS, AbuElmagd M, Al-Qahtani M. Human Wharton's Jelly Stem Cell (hWJSC) Extracts Inhibit Ovarian Cancer Cell Lines OVCAR3 and SKOV3 in vitro by Inducing Cell Cycle Arrest and Apoptosis. Front Oncol. 2018;8:592. 
19. Gauthaman K, Yee FC, Cheyyatraivendran S, Biswas A, Choolani M, Bongso A. Human umbilical cord Wharton's jelly stem cell (hWJSC) extracts inhibit cancer cell growth in vitro. J Cell Biochem. 2012;113(6):2027-39.

20. Santamaria X, Massasa EE, Taylor HS. Migration of cells from experimental endometriosis to the uterine endometrium. Endocrinology. 2012;153(11):5566-74.

21. Merdad A, Karim S, Schulten HJ, Dallol A, Buhmeida A, Al-Thubaity F, Gari MA, Chaudhary AG, Abuzenadah AM, Al-Qahtani MH. Expression of matrix metalloproteinases (MMPs) in primary human breast cancer: MMP-9 as a potential biomarker for cancer invasion and metastasis. Anticancer Res. 2014;34(3):1355-66.

22. Lu LS, Chen L, Ding WX, Li K, Wu JJ. Elevated expression of both MDR1 and MMP-2 genes in metastasized lymph node of invasive ductal breast cancer. Eur Rev Med Pharmacol Sci. 2012;16(15):2037-43.

23. Egeblad M, Werb Z. New functions for the matrix metalloproteinases in cancer progression. Nat Rev Cancer. 2002;2(3):161-74.

24. Hockenbery DM, Oltvai ZN, Yin XM, Milliman CL, Korsmeyer SJ. Bcl-2 functions in an antioxidant pathway to prevent apoptosis. Cell. 1993;75(2):241-51.

25. Chelluboina B, Klopfenstein JD, Gujrati M, Rao JS, Veeravalli KK. Temporal regulation of apoptotic and anti-apoptotic molecules after middle cerebral artery occlusion followed by reperfusion. Mol Neurobiol. 2014;49(1):50-65.

\section{Tables}

Table 1. The sequences and characteristics of primers used for detection of genes mRNA expression.

\begin{tabular}{|c|c|c|c|}
\hline Genes & Primers sequences & $\mathrm{T}_{\mathrm{m}}$ & Products size \\
\hline \multirow[t]{2}{*}{$B A X$} & F-CCCGAGAGGTCTTTTTCCGAG & $60^{\circ} \mathrm{C}$ & \multirow[t]{2}{*}{$155 \mathrm{bp}$} \\
\hline & R-CCAGCCCATGATGGTTCTGAT & $60^{\circ} \mathrm{C}$ & \\
\hline \multirow[t]{2}{*}{$B C L-2$} & \multirow{2}{*}{$\begin{array}{c}\text { F-GATGGGATCGTTGCCTTATG } \\
\text { R-GCGGAACACTTGATTCTGG }\end{array}$} & $56^{\circ} \mathrm{C}$ & \multirow[t]{2}{*}{$223 \mathrm{bp}$} \\
\hline & & $56^{\circ} \mathrm{C}$ & \\
\hline \multirow[t]{2}{*}{ Cas9 } & \multirow{2}{*}{$\begin{array}{c}\text { F-GCAGGCTCTGGATCTCGGC } \\
\text { R-GCTGCTTGCCTGTTAGTTCGC }\end{array}$} & $62^{\circ} \mathrm{C}$ & \multirow[t]{2}{*}{152 bp } \\
\hline & & $62^{\circ} \mathrm{C}$ & \\
\hline \multirow[t]{2}{*}{$S M A C$} & \multirow{2}{*}{$\begin{array}{l}\text { F-CAGAGGAGGAAGATGAAGTGTG } \\
\text { R-GCGGTTATAGAGGCCTGATCTG }\end{array}$} & $55^{\circ} \mathrm{C}$ & \multirow[t]{2}{*}{$196 \mathrm{bp}$} \\
\hline & & $56^{\circ} \mathrm{C}$ & \\
\hline \multirow[t]{2}{*}{ SURVIVIN } & F-CCCTTTCTCAAGGACCACCG & $52^{\circ} \mathrm{C}$ & \multirow[t]{2}{*}{$172 \mathrm{bp}$} \\
\hline & R-GTTCCTCTATGGGGTCGTCA & $51^{\circ} \mathrm{C}$ & \\
\hline \multirow[t]{2}{*}{ ACTIN } & F-AGAGCTACGAGCTGCCTGAC & $61^{\circ} \mathrm{C}$ & \multirow[t]{2}{*}{$184 \mathrm{bp}$} \\
\hline & R-AGCACTGTGTTGGCGTACAG & $60^{\circ} \mathrm{C}$ & \\
\hline
\end{tabular}




\section{Competing Interests}

The authors declare no conflicts of interest.

\section{Figures}
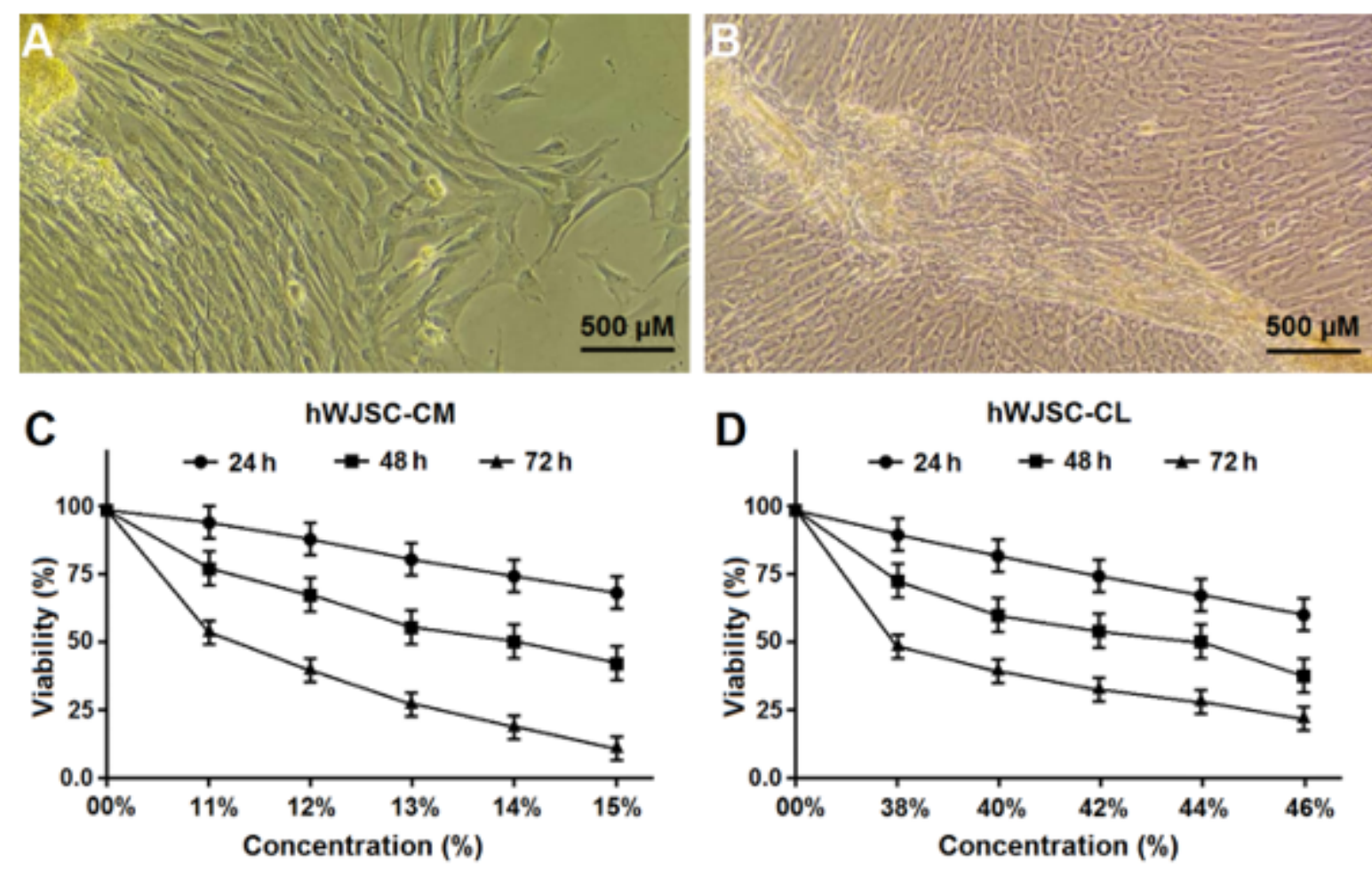

Figure 1

The inverted phase-contrast images from primary cultured hWJSCs, which shows the short fibroblasts characteristic (A) in early passages and long fibroblasts characteristic (B) in later passages (A). The HT29 cancer cells viability treated conditioned medium (C) and cell lysate (D) at 24-72 h. The results of the three experiments are presented as mean and SD values. 

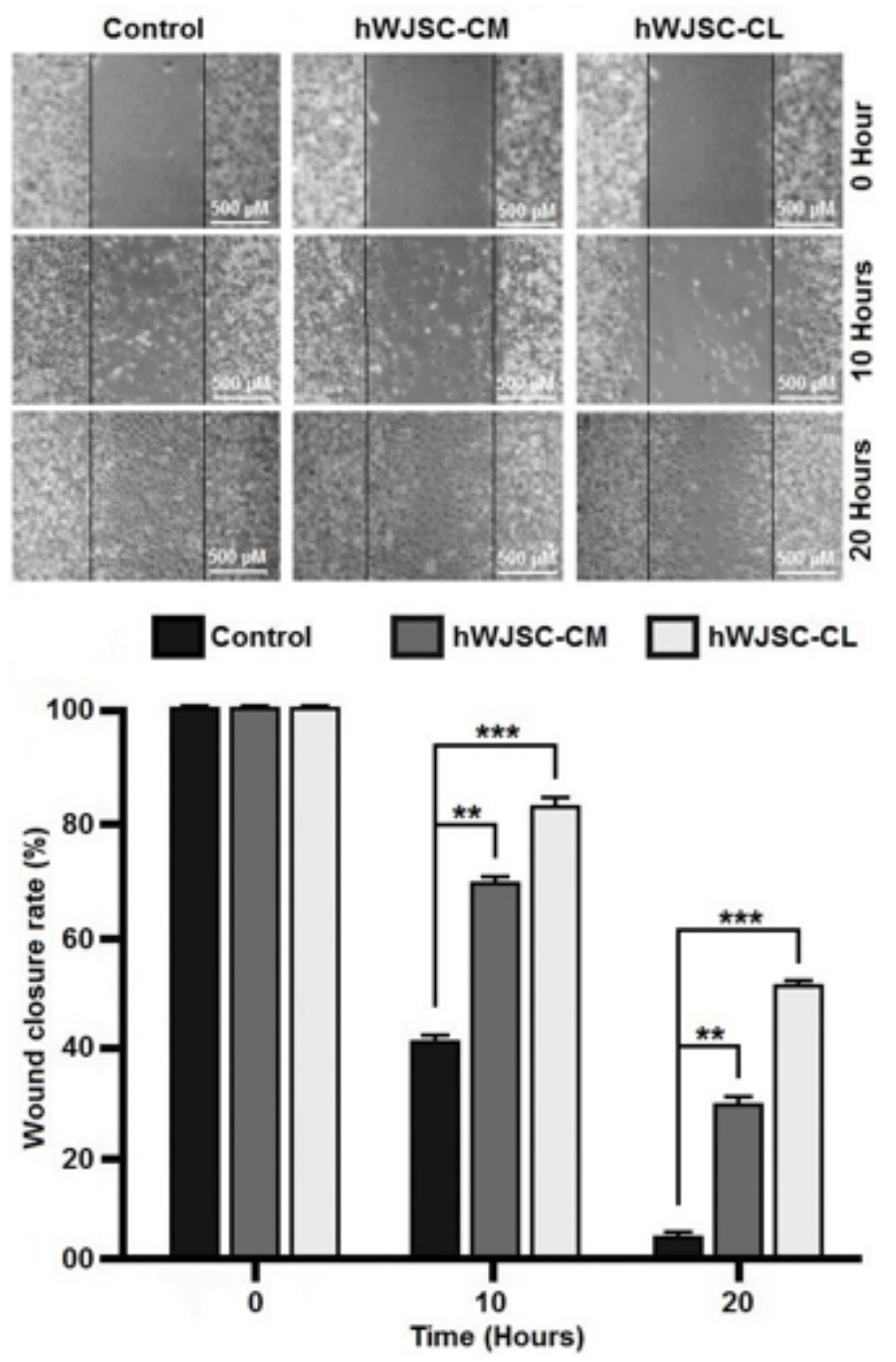

Figure 2

The inverted phase-contrast images from the effects of conditioned medium and cell lysate on migration of HT-29 cancer cells assessed using wound healing assay. The migration of cancer cells significantly decreased following treatment with conditioned medium and cell lysate. (hWJSC-CM: hWJSC conditioned medium, and hWJSC-CL: hWJSC cell lysate). 

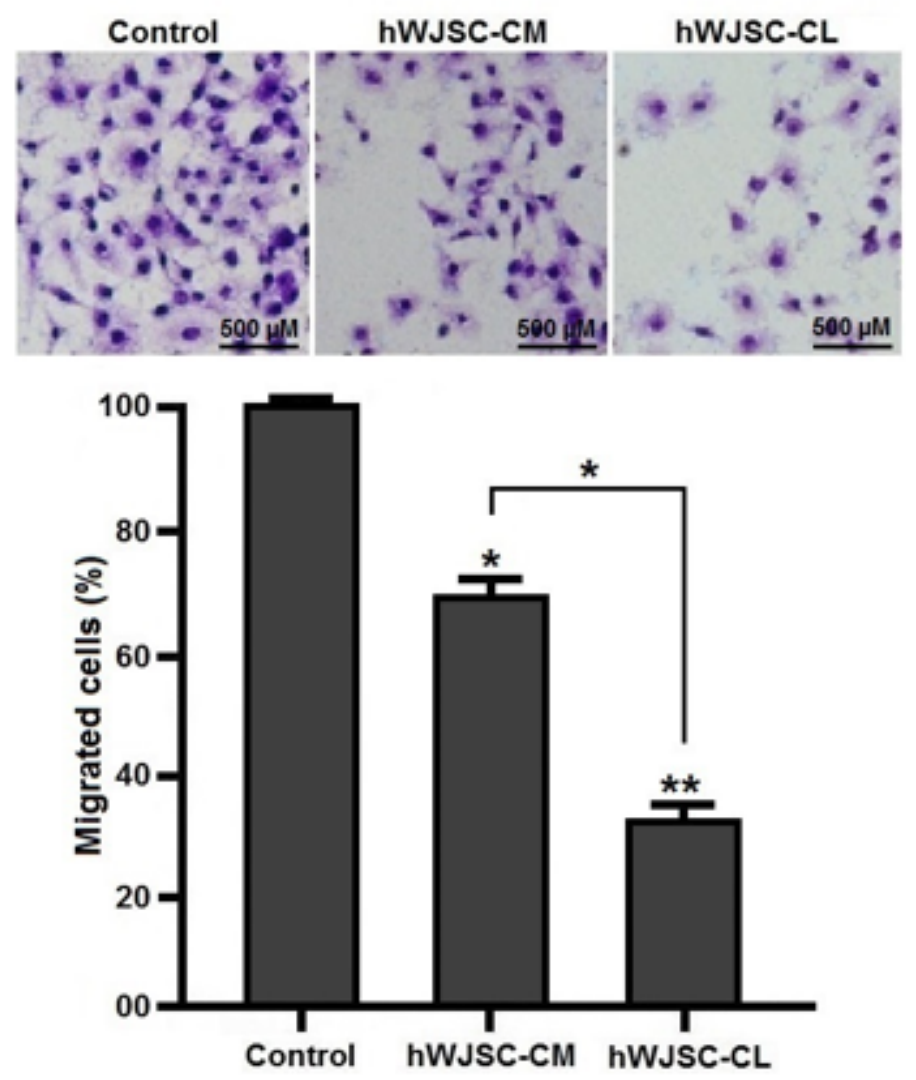

Figure 3

The inverted phase-contrast images from the effects of conditioned medium and cell lysate on invasion of HT-29 cancer cells assessed using transwell migration assays. The invasion of cancer cells significantly decreased following treatment with conditioned medium and cell lysate. (hWJSC-CM: hWJSC conditioned medium, and hWJSC-CL: hWJSC cell lysate). 

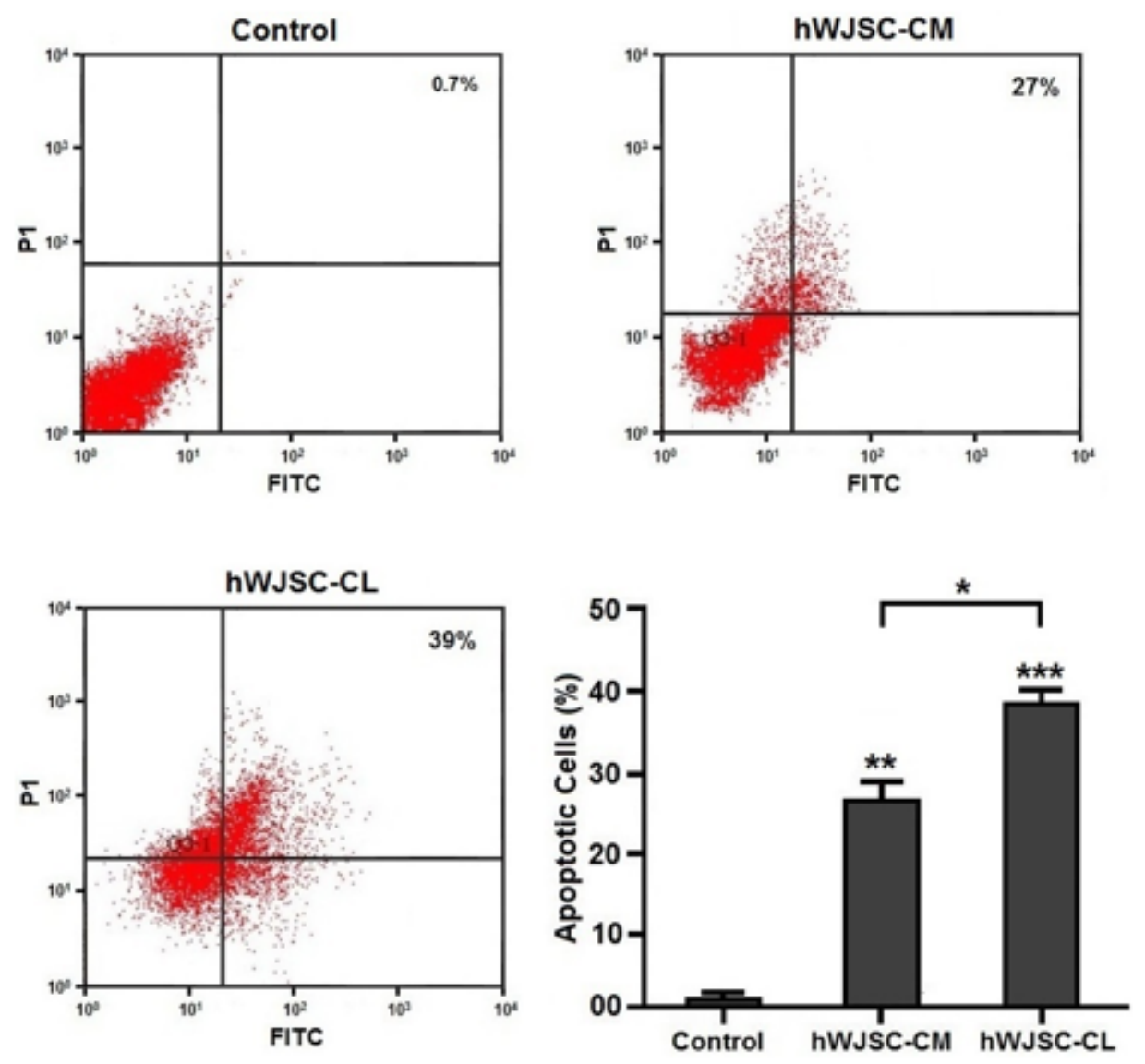

\section{Figure 4}

The effects of conditioned medium and cell lysate on apoptosis of HT-29 cancer cells. The apoptosis of cancer cells significantly increased following treatment with conditioned medium and cell lysate, as compared with untreated controls. (hWJSC-CM: hWJSC conditioned medium, and hWJSC-CL: hWJSC cell lysate). 

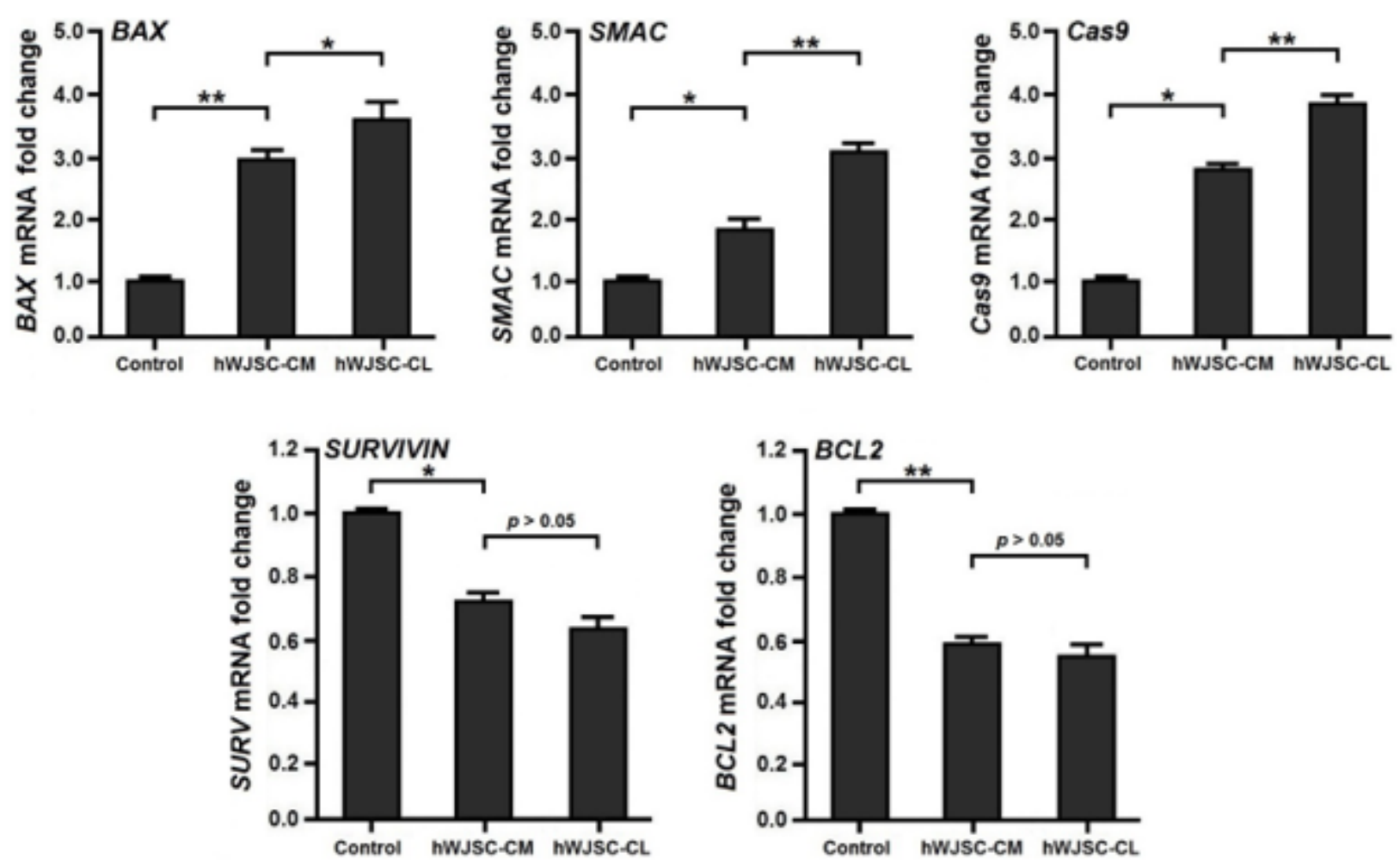

Figure 5

The mRNA expression analysis of apoptosis related BAX, SMAC, Cas9, SURVIVIN, and BCL-2 genes by qRT-PCR following the treatment with conditioned medium and cell lysate. The mRNA expression of BAX, SMAC, Cas9 in HT-29 cancer cells significantly increased as well as mRNA expression of BCL-2, and SURVIVIN significantly decreased following treatment with conditioned medium and cell lysate, as compared with untreated controls. (hWJSC-CM: hWJSC conditioned medium, and hWJSC-CL: hWJSC cell lysate). 